\title{
Archives
}

\section{La Division de la gestion de documents et des archives de l'Université de Montréal : un regard d'outre-Atlantique}

\section{PAUL SERVAIS}

Volume 46, numéro 2, 2017

$50^{\mathrm{e}}$ anniversaire de la DGDA

URI : https://id.erudit.org/iderudit/1040388ar

DOI : https://doi.org/10.7202/1040388ar

Aller au sommaire du numéro

Éditeur(s)

Association des archivistes du Québec (AAQ)

ISSN

0044-9423 (imprimé)

2369-9256 (numérique)

Découvrir la revue

Citer cet article

Servais, P. (2017). La Division de la gestion de documents et des archives de l'Université de Montréal : un regard d'outre-Atlantique. Archives, 46(2),

151-161. https://doi.org/10.7202/1040388ar
Résumé de l'article

Après avoir brièvement souligné les caractéristiques partagées et spécifiques inhérentes à un service d'archives d'université, ce texte met en lumière le rôle de la Division de la gestion de documents et des archives de l'Université de Montréal comme modèle, celui qu'elle joue comme incubateur ou pépinière d'archivistes et celui qu'elle assume comme moteur de la réflexion pour la communauté archivistique internationale. 


\section{ANNIVERSAIRE DE LA DGDA}

\section{La Division de la gestion de documents et des archives de l'Université de Montréal: un regard d'outre-Atlantique}

\section{PAUL SERVAIS}

Professeur émérite, Université catholique de Louvain

\section{INTRODUCTION}

Évaluer le rayonnement international d'une institution locale ou régionale, même importante, est une tâche particulièrement complexe. Elle suppose I'utilisation de nombreux indicateurs qui touchent aussi bien aux choix des secteurs d'investissement qu'aux fonctionnements ou aux modalités de partage des expériences.

Le point de vue que j'adopterais dans ce travail d'évaluation sera triplement extérieur:

- celui d'un responsable européen de service d'archives d'université travaillant dans un contexte culturel, organisationnel, légal et réglementaire différent;

- celui d'un enseignant en archivistique; 
- celui d'un bénéficiaire des atouts de l'observatoire de l'archivistique francophone que sont devenues au fil des ans les Journées des Archives de l'Université catholique de Louvain (UCL).

Je procéderai en plusieurs temps. Je rappellerai d'abord les spécificités $d^{\prime}$ 'un service d'archives universitaire. Puis, j'évoquerai successivement le service rendu à l'organisation, la participation aux missions de l'université et l'action des membres de la Division de la gestion de documents et des archives de I'Université de Montréal (DGDA), notamment pour ce qui est de la communication. Je terminerai en tentant d'accentuer ce qui m'apparaît comme les lignes de force les plus importantes du rayonnement international de la DGDA.

L'ensemble de cette analyse s'appuiera avant tout sur les constats effectués par deux de mes collègues, Cathy Schoukens et Evelyne Vandevoorde, lors d'un stage à la DGDA (2003). II s'appuiera ensuite sur les très nombreuses rencontres que j'ai pu avoir avec mes collègues de la DGDA, à Montréal ou en dehors du Québec, au cours des quinze dernières années.

\section{LES SPÉCIFICITÉS D'UN SERVICE D'ARCHIVES D'UNIVERSITÉ}

Tout service d'archives assure globalement trois missions que Claude Minotto formulait de la manière suivante dans un entretien accordé à la revue Forum en 2000: "Un service comme le nôtre a trois fonctions principales: conservation, traitement et diffusion des archives. » (Sauvé) En ce sens, il ne diffère pas de n'importe quel autre service de gestion des archives et ses impératifs sont les mêmes que partout ailleurs. Il y a déjà un moment, nous avions formulé ce défi à Louvain de la manière suivante: «fournir l'information pertinente, en temps utile aux personnes autorisées ».

Cependant, à y regarder de plus près, l'environnement spécifique d'une institution universitaire place le service des archives dans une position particulière. II n'est pas seulement un soutien à la gestion de l'administration universitaire, sous toutes ses facettes, et le garant de la conservation adéquate de la mémoire institutionnelle consignée. II ne se limite pas non plus à une expertise technique exceptionnelle en matière de gestion des atouts et des risques impliqués par l'explosion 
des nouvelles technologies dans la création, la gestion et la conservation des documents produits et reçus par l'institution. II vient également en support, voire est partie prenante, aux trois missions classiquement reconnues à l'université: I'enseignement, la recherche et le service à la société dans son domaine spécifique d'action, l'archivistique. Par le fait même, il est amené à constituer un service scientifique de logistique. On est donc fondé à attendre de lui qu'il contribue à l'action et au rayonnement de l'université tout entière, que ce soit par sa participation à l'enseignement, par le développement de programmes de recherche ou encore par la mise au service du milieu de son expérience, de sa réflexion, voire de sa logistique.

Concrètement, comme partie intégrante d'une institution à l'histoire souvent longue, le service des archives d'une université est ainsi responsable de la constitution d'un véritable patrimoine institutionnel. ॥ s'agit bien sûr d'abord de collecter, conserver et préserver des documents, anciens, précieux, voire fondateurs, qui n'ont plus de valeur immédiate, opérationnelle, mais une valeur de témoignage. Les membres du service des archives peuvent ainsi être régulièrement ou ponctuellement mobilisés à des fins de communication interne ou externe, voire dans l'objectif de perpétuer une identité spécifique ou de soutenir une véritable culture d'entreprise. II peut également s'agir de constituer les archives du futur, par exemple en suscitant des témoignages d'acteurs de l'institution universitaires afin de documenter non seulement les grands moments de la vie institutionnelle, mais également son quotidien. Enfin, ces documents peuvent également être informationnels et contribuer à une meilleure connaissance du patrimoine matériel, immobilier ou mobilier, là aussi à des fins de communication et de valorisation, sans que les préoccupations de gestion soient nécessairement absentes. Le cas de l'expansion et de la superposition de multiples réseaux - eau, gaz, électricité, informatique (dans ses nombreuses déclinaisons) - au cours du dernier siècle en constitue un bon exemple, avec la multiplicité des questions de maintenance, voire de remplacement, que cette situation suscite.

Pour leur participation à la mission d'enseignement de l'institution au service de laquelle il se trouve, il s'agit alors, pour le service d'archives, de mettre ses ressources documentaires à la disposition des enseignants et de leurs activités, qu'il s'agisse d'histoire, d'archivistique ou de sciences de la documentation. II s'agit aussi de prendre part directement à I'enseignement en accueillant des stagiaires, en encadrant des travaux 
ou des thèses, en intervenant dans certains enseignements, par exemple sous la forme de conférences ou de présentations, voire en assumant pleinement la responsabilité d'un cours ou d'un séminaire. De manière naturelle, le service d'archives est également, dans son domaine d'expertise, partie prenante de la mission de recherche de l'université. Ce qui se concrétise par une production scientifique à la fois en recherche fondamentale et en recherche appliquée, diffusée aussi largement que possible et soumise à la discussion de la communauté des archivistes, le cas échéant par l'organisation d'événements scientifiques, la participation à des organismes de réflexion, la publication d'études et de travaux.

En outre, un service d'archives d'université est, bien souvent, sollicité pour participer à la mission de service à la société qu'assume l'institution à laquelle il appartient, que ce soit par le conseil, le soutien, la mise à disposition d'expertise, l'accueil et le traitement de fonds spécifiques à un milieu ou un domaine, etc...

Globalement enfin, chacune de ces facettes d'activité devrait contribuer, de manière cohérente et coordonnée au rayonnement de l'université ellemême et, d'une manière ou d'une autre et suivant des modalités précises et différenciées, constituer un critère d'évaluation, une pierre de touche, de son rayonnement propre. Examinons dès lors brièvement ce qu'il en est pour la DGDA de I'Université de Montréal.

\section{LA DGDA ET LE SERVICE À L'ORGANISATION}

Quel que soit le contexte institutionnel ou organisationnel, le cœur de métier d'un service d'archives est de répondre aux attentes explicites, et parfois implicites, de l'entité au sein de laquelle il est intégré. En ce sens, la DGDA, ne présente pas nécessairement une originalité majeure: elle collecte, évalue, conserve, préserve et communique. Cependant, pour un regard européen, singulièrement celui de membres des archives de l'UCL effectuant un stage à la DGDA (Schoukens et Vandevoorde, 2003) un certain nombre d'éléments ont particulièrement frappé, notamment en comparaison avec la situation de leur service d'origine ou celle d'autres services européens similaires.

Dans leur rapport, ils ont d'abord mentionné la fonction de «greffe institutionnel » assumée par la DGDA, allant de pair avec une intégration dans la structure administrative et les organes de gestion de l'institution. 
La mise en place de procédures formelles et la formulation de règles de traitements des documents ou la constitution de calendriers de conservation ont constitué un second constat majeur de leur stage. La politique de formation des unités de production documentaire tout comme le choix d'utiliser les instruments de communication interne pour informer les membres de l'université sur les missions et réalisations de la DGDA ont retenu toute leur attention. Mais la contribution de la DGDA à la préservation, à l'enrichissement et à la valorisation du patrimoine institutionnel n'en a pas pour autant été occultée.

À bas bruit, sans gros investissement, sans nécessairement de volonté très explicite, ces différentes modalités de fonctionnement de la DGDA ont constitué le socle d'une première modalité de rayonnement, plutôt informelle, au-delà du périmètre de l'université et des frontières de la province. Comme modèle d'inspiration, elles ont constamment été en point de mire de l'action des Archives de I'UCL, justifiant par exemple l'organisation de réunions de travail entre les responsables de l'administration de l'UCL et des responsables, passé et présent, de la DGDA, à l'occasion d'un passage à Louvain-la-Neuve.

\section{LA DGDA ET LES MISSIONS DE L'UNIVERSITÉ}

Pour l'observateur extérieur, des trois missions classiques attribuées à I'Université: enseignement, recherche, service à la société, c'est d'abord I'enseignement qui retient l'attention. C'est que l'Université de Montréal comporte à la fois un Département d'histoire, grand utilisateur des services d'archives, et surtout un programme en trois niveaux (certificats de $1^{\text {er }}$ cycle, maîtrise, doctorat) d'archivistique intégré au sein de l'École de bibliothéconomie et des sciences de l'information (EBSI).

De manière pratiquement naturelle, la DGDA a dès lors pris en charge ou contribué à un ensemble de tâches d'enseignement, qui lui offrait autant de possibilité de partages d'expériences et de confrontations au regard neuf d'étudiants de différents niveaux. C'est d'abord l'accueil des stagiaires de l'EBSI qui a été relevé, de même que le prêt de documents au laboratoire d'archivistique de I'EBSI. Ont été soulignées ensuite la prise en charge de conférences à destination des étudiants ou encore les interventions lors de la présentation orale des travaux d'étudiants en archivistique au niveau de la maîtrise. Enfin, et il ne s'agit pas du moindre 
des investissements des membres de la DGDA, on a identifié un certain nombre de cours du programme d'archivistique régulièrement assumé par des membres de la DGDA.

Étroitement liée à la mission d'enseignement, la mission de recherche est également prise en charge par la DGDA, qu'il s'agisse d'une réflexion sur les fondements de l'archivistique, d'une recherche d'archivistique appliquée ou d'une recherche doctorale portant sur la contribution des stages à la formation des archivistes (Rousseau, 1997). La proximité de I'EBSI, de même que l'appartenance de plusieurs membres de la DGDA au Groupe interdisciplinaire de recherche en archivistique (GIRA) ${ }^{1}$, ont sans doute été de nature à faciliter cet investissement. Pour ce qui est d'évaluer le rayonnement international de la DGDA, c'est sans doute le segment d'activité le plus important ou, en tout cas, le plus visible. II suffit pour s'en convaincre d'interroger la base de données bibliographique du Portail International Archivistique Francophone (PIAF)². Même s'il s'agit d'une bibliographie collaborative et si les contributeurs québécois y jouent un rôle majeur, il est frappant d'y retrouver nombre de publications importantes des membres de la DGDA. Au total, on en dénombre plus de 80, parmi lesquelles on retrouve les noms de Carol Couture, Jean-Yves Rousseau, Claude Minotto, Diane Baillargeon, mais aussi Denys Chouinard, Taik Bourhis, Joachim Roy, etc. Ces suggestions de lecture reprennent notamment un grand classique largement diffusé, réédité et utilisé : Les fondements de la discipline archivistique, par JeanYves Rousseau, et Carol Couture ${ }^{3}$. On y retrouve également des études très techniques, par exemple, I'article Note de recherche. La reproduction de photographies à l'aide d'une caméra planéitaire par Joachim Roy et Jean-Yves Rousseau ${ }^{4}$, et, sous la plume de Taïk Bourhis, I'article Le projet Hippocampe: mise en place d'un système de gestion électronique de documents à I'Université de Montréal ${ }^{5}$. II y a aussi l'ouvrage toujours très sollicité de Michel Champagne et Denys Chouinard intitulé Le traitement $d^{\prime} u n$ fonds d'archives: ses documents historiques ${ }^{6}$.

La consultation des catalogues en ligne de bon nombre de bibliothèques universitaires, de bibliothèques généralistes ou de bibliothèques disciplinaires d'archivistique ne fait que confirmer ce constat: les publications des membres de la DGDA figurent en bonne place au niveau international, parmi un ensemble imposant de publications québécoises, ce qui témoigne de la vitalité de la discipline. 
Quant à la mission de service à la société, elle peut sembler plus naturellement locale ou régionale, donc moins susceptible de constituer un élément d'évaluation du rayonnement international de la DGDA. De fait, il $s^{\prime}$ agit aussi bien d'assistance à la recherche, que d'accueil et de traitement de fonds privés ainsi que de participations aux activités de comités ou souscomités interinstitutionnels ou régionaux, tel le Groupe d'archivistes de la région de Montréal $(G A R M)^{7}$. L'impact international de la DGDA est plus évident lors de la participation aux activités des associations professionnelles. Contribuer au fonctionnement de la revue de l'Association des Archivistes du Québec, Archives, signifie en fait soutenir le rayonnement d'une revue appréciée au niveau international et en favoriser le développement. Là aussi les répertoires bibliographiques ou les bibliothèques disciplinaires en constituent un témoignage éloquent. Même s'il est, cette fois, indirect, le rayonnement de la DGDA à l'international en est confirmé.

\section{LA DGDA, UNE PRÉSENCE À L'INTERNATIONAL}

La présence des membres de la DGDA à l'international, avant, pendant ou après leur passage à la DGDA constitue sans doute le dernier élément d'appréciation d'un rayonnement international. En effet, journées d'étude, conférences, colloques et séminaires sont les lieux privilégiés d'échange direct d'informations, de partage de pratiques, de mises en commun de réflexions. Ils constituent un élément essentiel de formation continue et la part qu'y prennent ou y ont pris les membres de la DGDA, à différents moments de leur carrière professionnelle, est impressionnante, qu'ils agissent comme intervenants ou comme animateurs et organisateurs.

Un premier exemple de cette implication pourrait certainement être la présence active qu'ils ont assurée aux Journées des Archives de l'Université catholique de Louvain ${ }^{8}$. Organisées depuis 2000, ces Journées sont petit à petit devenues un rendez-vous de l'archivistique et des archivistes francophones de Belgique, de France, de Suisse et du Québec. Les membres de la DGDA ont constamment eu à cœur d'y contribuer. On peut ainsi noter les contributions de Carol Couture, Claude Minotto ou Diane Baillargeon, parfois de manière simultanée, lors de différentes éditions et sur des thématiques tantôt appliquées, tantôt fondamentales. Ainsi, en 2012, Diane Baillargeon a présenté «Les réseaux d'acquisition d'archives privées au Québec» tandis que Carol Couture nous initiait à «L'approche des archives personnelles à Bibliothèque et Archives 
nationales du Québec ». En 2010, Diane Baillargeon était déjà mise à contribution en proposant sa réflexion sur « De la Déclaration québécoise à la Déclaration universelle sur les archives». En 2005, s'interrogeant sur les compétences attendues de l'archiviste, Claude Minotto plaide pour «La gestion des archives: Vision et pragmatisme», alors que Carol Couture tirait les conclusions de ces deux journées consacrées à la formation des archivistes. En 2004, on retrouve Diane Baillargeon pour la conférence inaugurale de la journée consacrée à la communication des archives, avec un exposé traitant de «De la déontologie du métier d'archiviste», alors qu'en 2002, c'est Carol Couture qui ouvre la Journée en présentant un exposé sur «La politique de gestion des archives: Une articulation des fonctions de l'archivistique contemporaine: Un aperçu des tendances et de l'évolution qui caractérisent la discipline archivistique et la profession d'archiviste en $2002 »$.

De manière plus générale, Claude Minotto attire très tôt l'attention sur l'importance de ces grandes rencontres internationales. II a lui-même joué un rôle important comme directeur exécutif du Congrès de Montréal en 1992, assurant ensuite le lien avec le congrès de Beijing en 1996, ce qui reflète sans doute son investissement et son parcours personnels à l'international. Deux articles en témoignent à loisir, d'une part Le Congrès international des archives: un outil pour le développement des archives en $1990^{\circ}$, ainsi que De Montréal à Beijing : impacts du Congrès international des archives en $1995^{10}$. Et la présence systématique de membres de la DGDA lors de grands congrès internationaux, par exemple les Conférences européennes des Archives (Florence en 2001, Genève en 2010) ou aux Congrès mondiaux du Conseil International des Archives (Vienne en 2004) ou à différentes conférences sectorielles ${ }^{11}$, confirme ce choix stratégique d'investissement à l'international, de participation à des réseaux internationaux, de visibilisation globale.

\section{CONCLUSION}

Au terme d'un trop court passage en revue, il serait déplacé de conclure de manière définitive quant au rayonnement international de la DGDA. Les indicateurs retenus devraient être complétés, leur analyse approfondie et des comparaisons avec d'autres institutions s'imposeraient pour pouvoir aller plus loin. 
Cependant, un certain nombre de constats apparaissent dès à présent, à commencer par le fait que la DGDA, entité au périmètre de responsabilités et d'actions bien délimité et à vocation avant tout locale, est bien présente dans le paysage international des archives, des archivistes et de l'archivistique. Qui plus est cette présence est multiforme quant à ses modalités - informelles/formelles, écrites/orales - comme pour ce qui est des domaines ou des thématiques à propos desquels elle se manifeste.

Ce qui pose de toute évidence la question du «pourquoi?». Sans démonstration fouillée et avec le regard extérieur qui est le mien, la phrase célèbre du juriste français Jean Bodin «il n'est de richesse que d'homme » me vient immédiatement à l'esprit. C'est que la DGDA, depuis sa constitution en service autonome rattaché au secrétariat général de l'université, semble avoir réussi à s'attacher des personnalités aux compétences diverses, mais d'envergure exceptionnelle, comme en témoignent leurs parcours. En effet, sans procéder à une analyse systématique des $C V$, on peut compter parmi ceux qui sont passés à la DGDA, ou qui s'y investissent toujours, pas moins de deux directeurs des archives nationales du Québec, des enseignants chevronnés, des chercheurs de haut niveau, dont un docteur, des archivistes recrutés après un parcours professionnel riche et diversifié, ou au contraire des archivistes au seuil d'une carrière, construisant leur expertise grâce à leur intégration dans une équipe performante et en constante démarche de perfectionnement, une équipe ouverte aux évolutions techniques, institutionnelles et sociétales.

Prête à relever les défis les plus contemporains, y compris pour ce qui est des représentations d'un métier et d'une profession, cette équipe est sans conteste le point fort de la DGDA, dans sa diversité et dans les complémentarités qu'elle peut mettre en œuvre. À un moment social où I'homme est souvent assimilé et réduit à une "ressource humaine » et à une ligne comptable dans la rubrique "Coûts», l'équipe de la DGDA me semble dès lors agir tantôt comme un incubateur, tantôt comme un révélateur, toujours comme un moteur de développement personnel et collectif. Son rayonnement international en donne un remarquable témoignage et offre à la communauté des archivistes un exemple inspirant à méditer, à imiter, à adapter. 


\section{NOTES}

1. Groupe interdisciplinaire de recherche en archivistique (GIRA): http://gira-archives.org/

2. Portail International Archivistique Francophone (PIAF) : http://www.piaf-archives.org/

3. ROUSSEAU, J.-Y. et COUTURE, C. (1994). Les fondements de la discipline archivistique. Saint-Foy, Québec: Presses de I'Université du Québec.

4. ROY, J. et ROUSSEAU, J.-Y. (1984). Note de recherche. La reproduction de photographies à l'aide d'une caméra planéitaire. Archives 16, 40-45.

5. BOURHIS, T. (2009-2010). Le projet Hippocampe: Mise en place d'un système de gestion électronique de documents à I'Université de Montréal. Archives 41(2), 135 161. Repéré à https://www.archivistes.qc.ca/revuearchives/vol41 2/41 2 bourhis.pdf

6. CHAMPAGNE, M. et CHOUINARD, D. (1987). Le traitement d'un fonds d'archives: ses documents historiques. La Pocatière - Montréal: Documentor - Université de Montréal, Secrétariat général, Service des Archives.

7. Groupe d'archivistes de la région de Montréal (GARM): https://archivisteraq.com/ groupe-dinteret/groupe-darchivistes-de-la-region-de-montreal-garm/

8. https://uclouvain.be/fr/decouvrir/archives/journees-des-archives.html

9. MINOTTO, C. (1990). Le Congrès international des archives: un outil pour le développement des archives. Archives 21, 59-75.

10. MINOTTO, C. (1995). De Montréal à Beijing: impacts du Congrès international des archives. Archives 26(4), 9-27. Repéré à https://www.archivistes.qc.ca/revuearchives/ vol26 4/26-4-minotto.pdf

11. Par exemple, lors des journées tenues à l'université de Lausanne les 30 et 31 octobre sur le thème des «Archives dans I'université», où Claude Minotto intervient sur le thème «Actualiser la mémoire institutionnelle de l'université ».

\section{BIBLIOGRAPHIE}

ROUSSEAU, J.-Y. (1997). Étude descriptive de la contribution du stage à la formation professionnelle initiale des archivistes du Québec de 1983 à 1994. (Thèse de doctorat, Université de Montréal). Repéré à https:// papyrus.bib.umontreal.ca/xmlui/bitstream/handle/1866/6758/these. html;jsessionid=1AD1E740C3D6C346D61EE244454A2031 
SAUVÉ, M.-R. (2000). Claude Minotto et la mémoire institutionnelle. La Division des archives négocie le virage technologique. Forum 35(2). Repéré à http://www.forum.umontreal.ca/numeros/2000 2001/ forum 0009 05/article11.html

SCHOUKENS, C. et VAN DE VOORDE, E. (2003). Rapport de stage à la Division des Archives de l'Université de Montréal (Document interne non publié). Louvain-la-Neuve, Belgique: Archives de I'Université de Louvain-la-Neuve. 